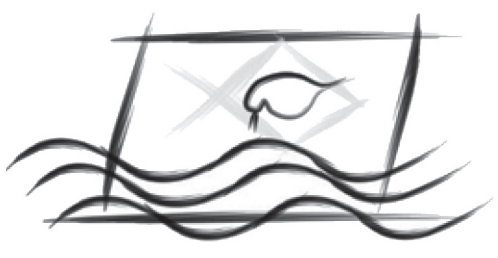

J. Braz. Soc. Ecotoxicol., v. 5, n. 1, 2010, 49-54

doi: $10.5132 /$ jbse.2010.01.008

ECOTOX - Brazil

\title{
Distribuição de Mercúrio Total em Tecido Muscular de Hypostomus affinis (Steindachner, 1877) e Hypostomus c.f. luetkini (Barlenger, 1887) do Rio Muriaé, Itaperuna - RJ
}

\author{
B. F. Araujo ${ }^{1 *}$, C. E. V. Carvalho ${ }^{*}$, D. R. Andrade ${ }^{2}$, R. S. Gomes ${ }^{1} \&$ C. T. Souza ${ }^{2}$ \\ ${ }^{1}$ Laboratório de Ciências Ambientais, Centro de Biociências e Biotecnologia, \\ Universidade Estadual do Norte Fluminense Darcy Ribeiro, Av. Alberto Lamego, 2000, \\ CEP 28015-602, Campos dos Goytacazes - RJ, Brasil \\ ${ }^{2}$ Laboratório de Zootecnia e Nutrição Animal, Centro de Ciências e Tecnologias Agropecuárias, \\ Universidade Estadual do Norte Fluminense Darcy Ribeiro, Av. Alberto Lamego, 2000, \\ CEP 28015-602,Campos dos Goytacazes - RJ, Brasil
}

(Received January 28, 2008; Accepted March 7, 2009)

\begin{abstract}
RESUMO
O presente trabalho teve como principais objetivos avaliar a qualidade do pescado através da determinação de concentrações de mercúrio total (HgT) no tecido muscular das espécies de cascudo Hypostomus affinis (Steindachner,1877) e Hypostomus c.f. luetkini (Barlenger,1887), coletados no Rio Muriaé, no município de Itaperuna - RJ e avaliar suas variações entre meses da estação seca e chuvosa. Para as determinações de HgT, foram coletados cinco peixes de cada espécie durante os meses de agosto de 2005 a janeiro de 2006. A espécie $H$. affinis apresentou concentrações médias menores que a espécie $H$. c.f. luetkini com exceção do mês de agosto. As maiores médias encontradas foram no mês de outubro para $H$. affinis $\left(33,4 \mathrm{ng} \cdot \mathrm{g}^{-1}\right.$ em peso úmido) e $H$. c.f. luetkini (39,5 ng. $\mathrm{g}^{-1} \mathrm{em}$ peso úmido). Em relação às variações mensais as duas espécies apresentaram as maiores concentrações de Hg no período de seca (agosto a setembro) em comparação ao período chuvoso (outubro a novembro). Todas as amostras analisadas apresentaram concentrações dentro do limite estabelecido pela ANVISA para peixes não carnívoros (500 ng. $\mathrm{g}^{-1} \mathrm{em}$ peso úmido).
\end{abstract}

Palavras-chave: mercúrio, Hypostomus, saúde pública, variações mensais, Rio Muriaé.

\section{ABSTRACT}

Total Mercury Distribution in Muscular Tissue of Hypostomus affinis (Steindachner, 1877) and Hypostomus c.f. luetkini (Barlenger, 1887) from Muriaé River, Itaperuna - RJ

With the purpose of evaluating the consumed fish quality, total mercury concentration were analyzed in muscle samples from two tropical freshwater fish (Hypostomus affinis Steindachner,1877 and Hypostomus c.f. luetkini, Barlenger,1887) from the Muriaé River, Itaperuna Municipality, in the North of Rio de Janeiro State. A secondary objective of the present study was to compare mercury concentration in these two species during dry and wet seasons. In order to achieve these goals, five organisms from each species were monthly collected between August 2005 and January 2006. H. affinis always presented lower total mercury average concentration when compared with H. c.f. luetkini with exception of August 2005 when an opposite trend was observed. The highest mercury concentrations were observed in October for both species (H. affinis $33.4 \mathrm{ng} . \mathrm{g}^{-1}$ in wet weight and H. c.f. luetkini $39.5 \mathrm{ng} . \mathrm{g}^{-1}$ in wet weight) during the beginning of dry season. There was a clear trend of higher $\mathrm{Hg}$ concentrations during the dry season when compared to the wet season probably related with the dilution effect caused by the increasing water discharges during the wet season. All analyzed samples presented total mercury concentrations lower than the maximum permitted concentration for human consumption established by ANVISA and WHO (500 ng. $\mathrm{g}^{-1}$ in wet weight), therefore no risk for human consumption was observed.

Keywords: mercury, Hypostomus, public health, seasonal variations, Muriaé River.

* Corresponding author: Beatriz Ferreira Araújo, e-mail: bfaraujo@yahoo.com.br; Carlos Eduardo Veiga de Carvalho, e-mail: carvalho@uenf.br 


\section{INTRODUÇÃO}

Dentre os metais pesados, o $\mathrm{Hg}$ é reconhecido como um dos poluentes com maior potencial tóxico por apresentar a capacidade de bioacumular-se e biomagnificar-se ao longo da cadeia trófica (Förstner; Wittmann, 1983).

A ingestão de mercúrio através do consumo de peixes é a principal via de contaminação do homem, o qual é capaz de acumular o metilmercúrio $(\mathrm{MeHg})$ que é a forma orgânica mais tóxica deste metal (Pfeiffer et al., 1992). O MeHg é uma substância lipossolúvel que possui uma tendência para se concentrar no tecido muscular dos peixes, ligando-se a grupos sulfidrilas de proteínas, sendo que cerca de $90 \%$ do $\mathrm{Hg}$ total disponível no tecido muscular está sob a forma de $\mathrm{MeHg}$ (Weis, 2004). A metilação de Hg é o passo mais importante para este elemento entrar na cadeia alimentar aquática (Dorea et al., 2006).

As espécies Hypostomus affinis e Hypostomus c.f. luetkini, da família Loricariidae, são peixes que se alimentam de detritos do fundo e pertencem à biocenose bentônica, participando da fase de pré-mineralização da matéria orgânica presente no substrato lodoso, facilitando sua decomposição pelos microorganismos e, em decorrência disso, acelerando a reciclagem de nutrientes e proporcionando a dinamização do metabolismo aquático (Azevedo, 1938). Em contrapartida, essas espécies de peixe podem absorver poluentes através de sua alimentação, liberando para a cadeia trófica e para a coluna d'água compostos que se encontravam indisponíveis no sedimento, como o MeHg. Os detritos consumidos por essas espécies possuem alto grau de celulose de acordo com a análise do conteúdo estomacal (Azevedo,1938), contudo estudos isotópicos delta-C13 e delta $\mathrm{N}-15$ demonstram que espécies de fundo não apresentaram especificidade quanto à fonte autotrófica (Benedito-Cecilio; Araújo-Lima,2002).

A região estudada possui um histórico de extração do ouro aluvionar, onde na década de 80 esse rio foi utilizado como rota alternativa para a atividade (Souza, 1994). Além disso, outra potencial fonte de $\mathrm{Hg}$ pode ser atribuída à utilização de fungicidas organomercuriais na agricultura, que ocorreu até o início dos anos oitenta quando foi proibido o emprego de compostos a base de Hg (Câmara,1990). Os objetivos do presente trabalho foram avaliar a qualidade do pescado através da determinação de concentrações de $\mathrm{HgT}$ no tecido muscular das espécies de $H$. affinis e H. c.f. luetkini e comparar suas concentrações entre as estações seca e chuvosa nas duas espécies do gênero Hypostomus. Tendo em vista que a atividade pesqueira na região de Itaperuna é representativa e permanente sendo as espécies da família Loricariidae altamente consumidas nessa região, torna-se importante realizar um estudo preliminar sobre a qualidade do pescado que é consumido (Souza et al., 2007)

\section{MATERIAL E MÉTODOS}

\section{Área de estudo}

O Rio Muriaé é um importante afluente da margem esquerda do Rio Paraíba do Sul, com aproximadamente $300 \mathrm{Km}$ de extensão, drenando uma área de $8.230 \mathrm{Km}^{2}$ (Vargas;
Damiance, 2003) por o fluxo médio de $2,2 \times 10^{-5} \mathrm{Km}^{3} / \mathrm{Km}^{2} /$ Seca e $7,9 \times 10^{-5} \mathrm{Km}^{3} / \mathrm{Km}^{2} /$ Cheia (ANA, 2006). O Rio Muriaé tem sua foz no Rio Paraíba do Sul confluindo com este no município de Campos dos Goytacazes, Norte do Estado do Rio de Janeiro (Figura 1).

\section{Amostragens}

As amostras foram obtidas mensalmente em um único ponto de coleta às margens do Rio Muriaé $\left(21^{\circ} 12^{\prime} 21,79^{\prime \prime} \mathrm{Se}\right.$ $\left.41^{\circ} 54^{\prime} 15,58^{\prime \prime} \mathrm{O}\right)$ no centro do município de Itaperuna, entre agosto de 2005 e janeiro de 2006. No total, 60 indivíduos foram considerados no presente estudo, conforme apresentado na Tabela 1. A biometria foi realizada em laboratório na UENF, onde de cada peixe foram retiradas amostras do tecido muscular as quais foram congeladas para análise de Hg. Foram utilizados cinco brancos para cada 25 amostras em triplicata. O procedimento para extração química de $\mathrm{Hg}$ total foi baseado na metodologia descrita por Bastos et al., (1998), onde alíquotas de $1 \mathrm{~g}$ de tecido muscular de peixe (massa úmida) foram transferidas para tubos de ensaio de vidro. Em seguida, foram adicionados $1 \mathrm{~mL}$ de $\mathrm{H}_{2} \mathrm{O}_{2} 30 \%$ (em gelo) e depois $3 \mathrm{~mL}$, de uma solução $\mathrm{H}_{2} \mathrm{SO}_{4 \text { (conc) }}: \mathrm{HNO}_{3 \text { (conc) }}(1: 1)$. Após essa etapa os tubos foram para o bloco digestor à $60^{\circ} \mathrm{C}$ até completa solubilização. Logo adicionou-se $5 \mathrm{~mL}$ de $\mathrm{KMnO}_{4} 5 \%$ e aqueceu-se à $60{ }^{\circ} \mathrm{C}$ em bloco digestor por 30 minutos. Após o resfriamento, as amostras foram tituladas com solução de cloreto de hidroxilamina $12 \%$ até o ponto de viragem e filtradas. Aferiu-se o volume a $20 \mathrm{~mL}$ utilizando-se água Mili-Q.

Todas as determinações de $\mathrm{Hg}$ em amostras de tecido muscular foram realizadas por ICP-AES da Varian (modelo Liberty II) com acessório de geração de vapor frio (VGA-77). A exatidão da metodologia analítica de determinação da concentração de $\mathrm{Hg}$ no tecido muscular por ICP-AES foi checada e confirmada através do uso de material certificado de referência - DORM - 1 (tecido muscular de Squalus acanthias), preparado pela "Marine Analytical Chemistry Standarts Programs", Canadá, analisado em triplicata em quatro testes. A concordância entre o resultado analítico do material de referência determinado e seu valor certificado foi de $98,92 \%$, com coeficiente de variação analítico inferior a $10 \%$.

O limite de detecção (LD) da técnica usada foi de 17,93 ng. $\mathrm{g}^{-1}$ em peso úmido. O cálculo seguiu a metodologia descrita por Skoog e Leary (1992) de acordo com a Equação 1:

$L D=\frac{(3 \times D P \text { branco })}{a}$

sendo: $D P$ branco representa o desvio padrão de um número significativo de brancos (40) e $a$ corresponde à inclinação da curva de calibração.

\section{RESULTADOS E DISCUSSÃO}

De maneira geral, as concentrações foram maiores para a espécie H.c.f. luetkini em relação à $H$. affinis, com exceção do mês de agosto (Tabela 1), porém as duas espécies do gênero Hypostomus apresentaram concentrações muito baixas de mercúrio total no tecido muscular, Cerca de $45 \%$ das amostras analisadas apresentaram valores abaixo do limite de detecção do aparelho, ou seja, menores que 17,93 ng.g ${ }^{-1}$. 

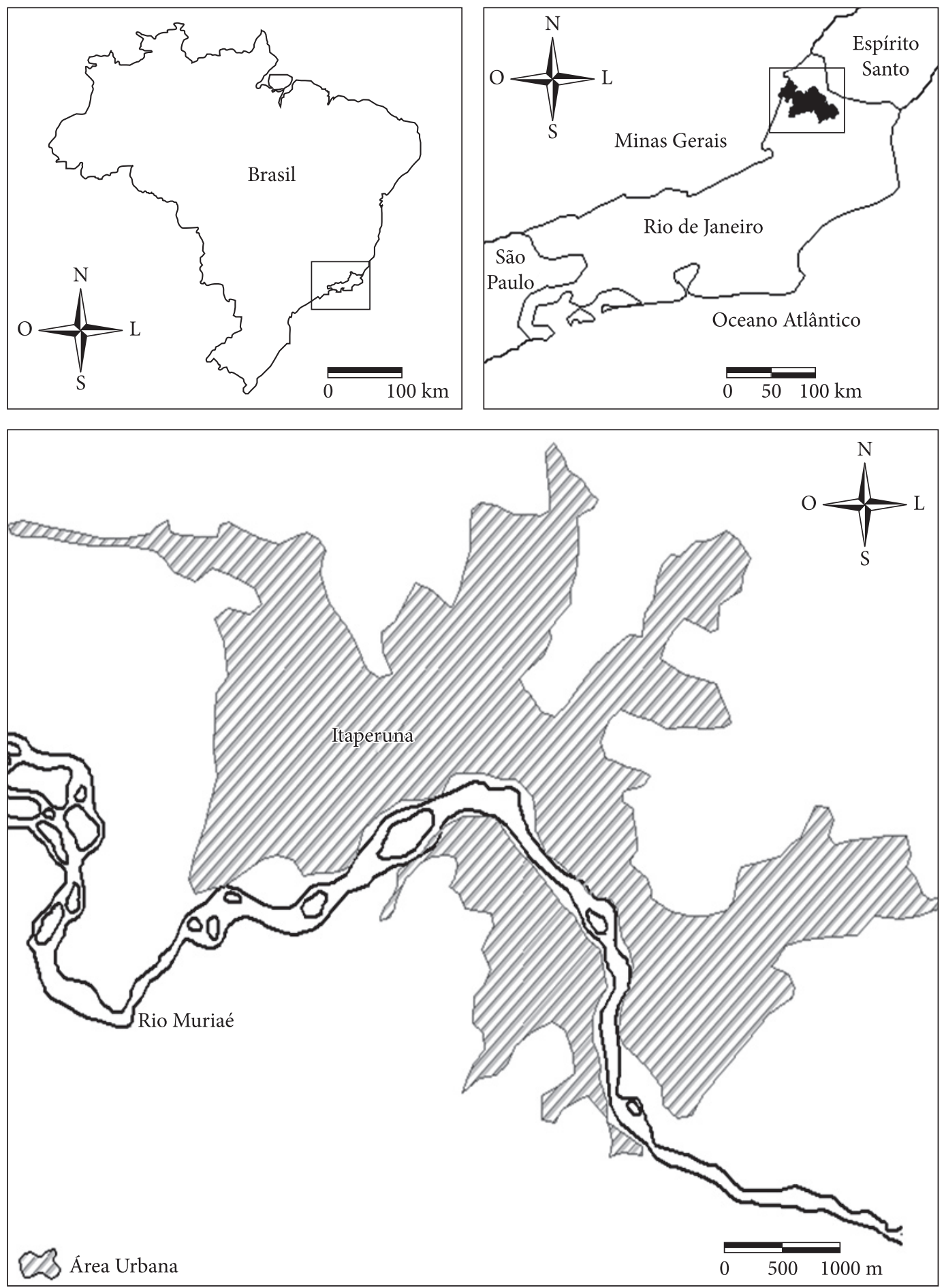

Figura 1 - Localização do rio Muriaé no município de Itaperuna, RJ.

Tabela 1 - Porte e concentração média de Hg em espécimes de H. affinis e H. c.f. luetkini coletados no Rio Muriaé entre agosto de 2005 e janeiro de 2006.

\begin{tabular}{|c|c|c|c|c|c|c|}
\hline \multirow[t]{2}{*}{ Mês } & \multirow[t]{2}{*}{$\mathrm{N}$} & \multicolumn{2}{|c|}{ H. affinis } & \multirow[t]{2}{*}{$\mathrm{N}$} & \multicolumn{2}{|c|}{ H. c.f. luetkini } \\
\hline & & Porte $(\mathrm{g}-\mathrm{cm})$ & $\mathrm{Hg}\left(\mu \mathrm{g} \cdot \mathrm{Kg}^{-1}\right)$ & & Porte $(\mathrm{g}-\mathrm{cm})$ & $\mathrm{Hg}\left(\mu \mathrm{g} \cdot \mathrm{Kg}^{-1}\right)$ \\
\hline Agosto & 5 & $167 \pm 20,1$ & $21,8 \pm 18,6$ & 5 & $109,5-19,1$ & $18,6 \pm 7,8$ \\
\hline Setembro & 5 & $149,2 \pm 20,1$ & nd & 5 & $93,92-16,5$ & $27,5 \pm 7,6$ \\
\hline Outubro & 5 & $226,6 \pm 20,1$ & $33,4 \pm 29,7$ & 5 & $99,2-15,3$ & $39,5 \pm 6,5$ \\
\hline Novembro & 5 & $226,9 \pm 22,8$ & nd & 5 & $87,2-22,8$ & $20,1 \pm 3,1$ \\
\hline Dezembro & 5 & $151,4 \pm 20,6$ & nd & 5 & $68-15,4$ & nd \\
\hline Janeiro & 5 & $172,8 \pm 20,6$ & nd & 5 & $64,7-13,6$ & $22,1 \pm 16,9$ \\
\hline
\end{tabular}


Para $H$. affinis, o maior valor encontrado foi no mês de outubro (33,4 ng. $\left.\mathrm{g}^{-1}\right)$ e o menores em dezembro e janeiro $\left(<17,93 \mathrm{ng} \cdot \mathrm{g}^{-1}\right)$. Da mesma forma H. c.f. luetkini apresentou a maior concentração em outubro $\left(39,5 \mathrm{ng} \cdot \mathrm{g}^{-1}\right)$ e a menor em

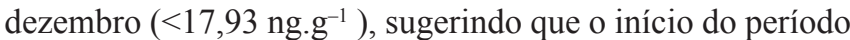
chuvoso influenciou a entrada do material particulado em suspensão através do escoamento superficial.

As relações de comprimento padrão vs concentração de $\mathrm{Hg}$ e peso vs. concentração de $\mathrm{Hg}$ para $H$. affinis e H. c.f. luetkini não foram significativas, apresentando baixos valores de $r^{2}$ (Figura 2). Estes resultados possivelmente foram influenciados pelo grande número de amostras terem apresentado concentrações abaixo do limite de detecção do aparelho.

As concentrações de mercúrio total para as duas espécies coletadas no Rio Muriaé, Hypostomus affinis e Hypostomus c.f. luetkini, durante os meses de agosto de 2005 a janeiro de 2006 apresentaram valores inferiores a $500 \mathrm{ng} . \mathrm{g}^{-1} \mathrm{em}$ peso úmido limite máximo para consumo humano em peixes detritívoros determinado pela ANVISA (Agência Nacional de Vigilância Sanitária, 1998) Portaria $n^{\circ} 685$, indicando que esse pescado não apresenta risco a saúde humana. Esses resultados são de relevante importância considerando que essas espécies são muito consumidas na cidade de Itaperuna.

As concentrações de Hg foram maiores no período de seca (agosto a outubro), em relação ao período chuvoso (novembro a janeiro) para ambas as espécies (Figura 3). No mês de outubro notou-se um aumento das concentrações de $\mathrm{Hg}$ nos peixes, período onde foi observada a menor pluviosidade, e no mês de janeiro percebe-se uma diminuição dessas concentrações de $\mathrm{Hg}$ nas amostras. Este resultado provavelmente foi influenciado
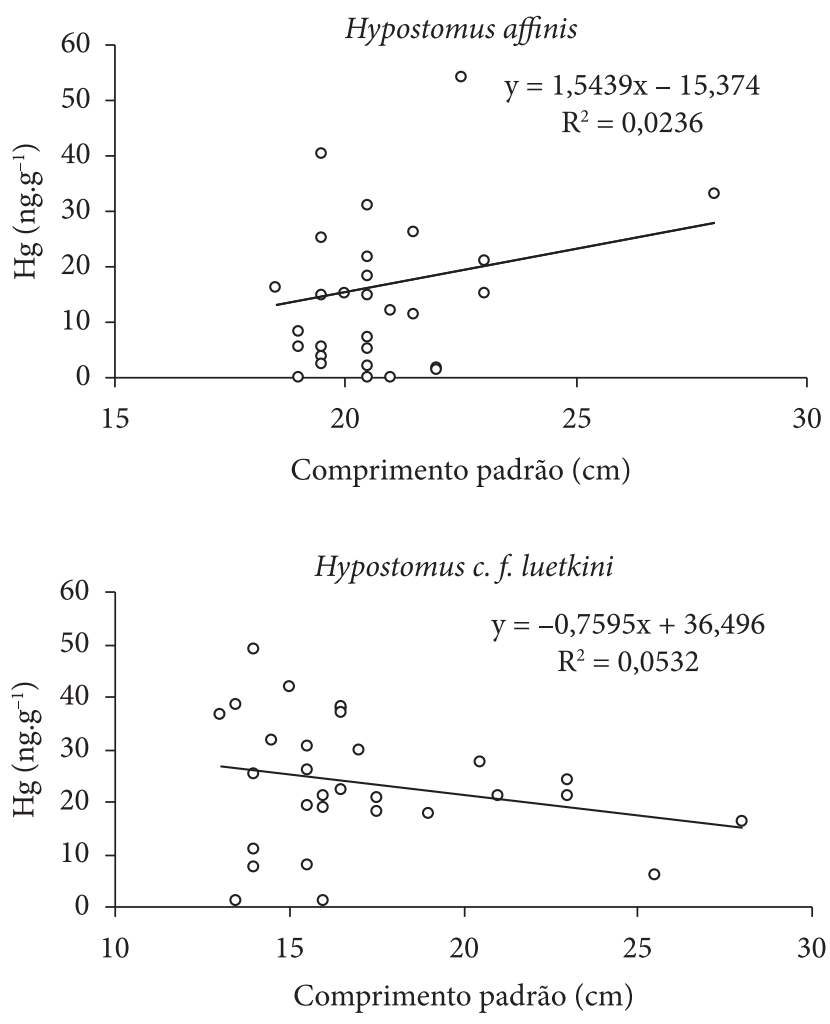

pela estação chuvosa, cujo efeito é o aumento do volume de água no rio, causando uma diluição do Hg presente na coluna d'água, diminuindo a disponibilidade desse elemento para a biota (Carvalho et al.,1999).

O processo de diluição de espécies metálicas já haviam sido anteriormente descrito para a região em estudo considerando $\mathrm{Hg}$ tanto como para outros elementos no Rio Paraíba do Sul e no Rio Muriaé (Almeida et al., 2007; Carvalho et al., 1999). O estudo realizado por Rodrigues (2003) no Rio Paraíba do Sul com a espécie de peixe Hoplias malabaricus reforça a hipótese. Outro fator, que poderia estar influenciando, seria a própria concentração do Hg na área estudada, uma vez que Almeida et al., (2007) encontrou baixas concentrações no material particulado em suspensão no mesmo rio.

No mês de outubro, as concentrações de Hg apresentaram aumento para ambas as espécies, que coincide com o início da estação chuvosa. As primeiras chuvas que caem em uma bacia de drenagem após um período de estiagem são geralmente enriquecidas em material particulado em suspensão que é o principal suporte geoquímico para poluentes metálicos. Além disso, no início do período chuvoso os rios ainda apresentam o seu volume de água reduzido, que ao receber este escoamento superficial mais enriquecido pelo $\mathrm{Hg}$ originário dos fungicidas (Câmara,1990) e resultante da precipitação atmosférica refletem em um pico de concentração (Almeida et al., 2007).

Os maiores valores de pluviosidade e vazão foram observados no mês de dezembro (240 $\mathrm{mm}$ e $140 \mathrm{~m}^{3}$, respectivamente). Os menores valores foram registrados em agosto (pluviosidade: 3,2 mm e vazão: $21,2 \mathrm{~m}^{3} \cdot \mathrm{s}^{-1}$ ), seguido do mês de outubro (pluviosidade: $10 \mathrm{~mm}$ e vazão: 21,6 $\mathrm{m}^{3} \cdot \mathrm{s}^{-1}$ ) (Figura 4).
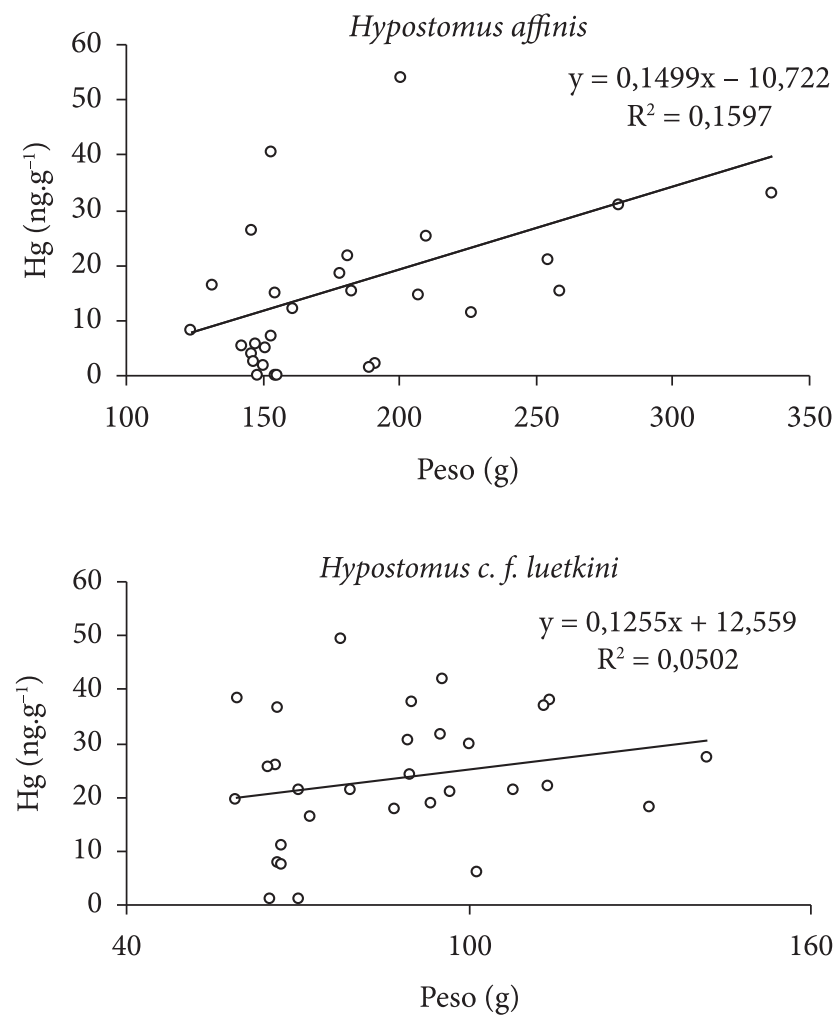

Figura 2 - Correlação do comprimento padrão e peso X concentração de $\mathrm{Hg}$ para as espécies H. affinis e H. c.f. luetkini. 
Tabela 2 - Tabela comparativa de concentração de Hg Total (ng. $\left.\mathrm{g}^{-1}\right)$ em espécies detritívoras em diferentes sítios de coleta.

\begin{tabular}{|c|c|c|c|c|}
\hline Sítio de coleta & Espécie & $\mathrm{N}$ & {$[\mathrm{Hg}]$ média ng.g ${ }^{-1}$} & Peso médio $(\mathrm{g})$ \\
\hline Rio Muriaé, $\mathrm{RJ}^{1}$ & Hypostomus affinis & 30 & $2,8-33,4$ & 183,2 \\
\hline Rio Muriaé, RJ & Hypostomus c.f. luetkini & 30 & $14,2-39,5$ & 187,1 \\
\hline Lagoa de Cima, $\mathrm{RJ}^{2}$ & Cyphocarax gilbert & 29 & $34,4-124,1$ & 48,9 \\
\hline Lagoa do Campelo, RJ & Cyphocarax gilbert & 09 & $47,9-78,1$ & 51,8 \\
\hline Rio Maroni, Guiana Francesa ${ }^{3}$ & Doras micropoeus & 08 & 1252 & 301 \\
\hline Rio Negro, $\mathrm{AM}^{4}$ & Potamorhina latior & 09 & $49,5-213,5$ & 194 \\
\hline
\end{tabular}

${ }^{\star 1}$ ) presente estudo; ${ }^{2}$ ) Souza, 2005; ${ }^{3}$ ) Alain et al., 2006; ${ }^{4}$ ) Dorea et al., 2006.

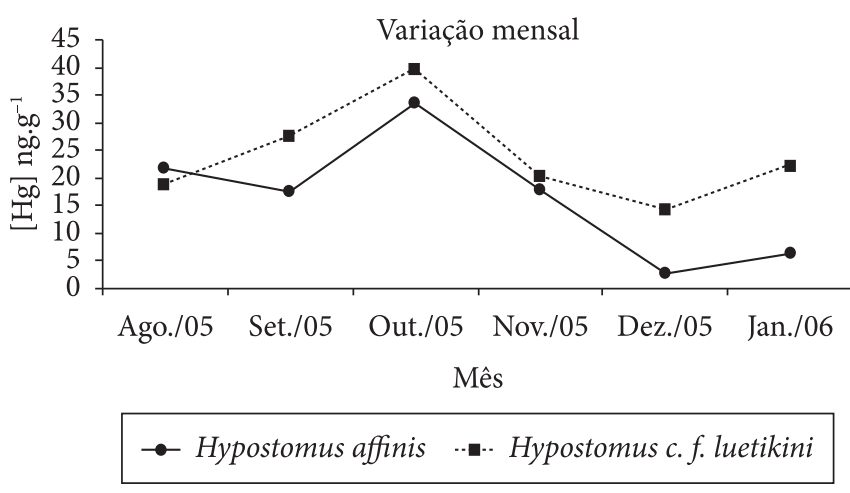

Figura 3 - Variação mensal das concentrações de Hg em H. affinis e H. c.f. luetkini entre os meses de agosto/05 a janeiro/06.

A alta pluviosidade promove uma ressuspensão do sedimento de fundo que tende a diluir a concentração do mercúrio ligado ao material particulado em suspensão (Almeida et al., 2007). A vazão durante o período de estudo se comportou de forma oposta a concentração de $\mathrm{Hg}$, ou seja, as menores vazões observadas, compreendidas no período de seca (agosto a outubro), representaram as maiores concentrações de Hg para ambas as espécies estudadas.

Considerando as diferenças sazonais entre as estações seca e chuvosa, as maiores concentrações de $\mathrm{Hg}$ foram observadas para ambas as espécies no período compreendido entre os meses de agosto a outubro (estação seca) em comparação ao período que envolve os meses de novembro a janeiro (estação chuvosa), o que indica que o aumento da pluviosidade e conseqüente vazão do rio estejam acentuando o efeito da diluição do mercúrio.

Espécies de hábito detritívoro podem apresentar diferentes taxas de acúmulo de mercúrio. Na Tabela 2 estão apresentados os dados de concentração de Hg total em espécies de hábito detritívoro em diversos estudos. Pode-se observar as variações nos níveis de mercúrio encontrados nas espécies estudadas, quando comparadas com as de outros trabalhos.

O Rio Muriaé já sofreu atividade garimpeira e intensa utilização de fungicidas organomercuriais nas décadas de 70 e 80 em sua bacia de drenagem, após esse período as atividades garimpeiras foram proibidas assim como a utilização de fungicidas mercuriais nas plantações de cana de açúcar. Apesar destas duas fontes pretéritas de mercúrio as concentrações observadas no presente estudo foram baixas quando comparadas a áreas contaminadas por $\mathrm{Hg}$, estando às concentrações próximas as de áreas livres de contaminação (Tabela 2).
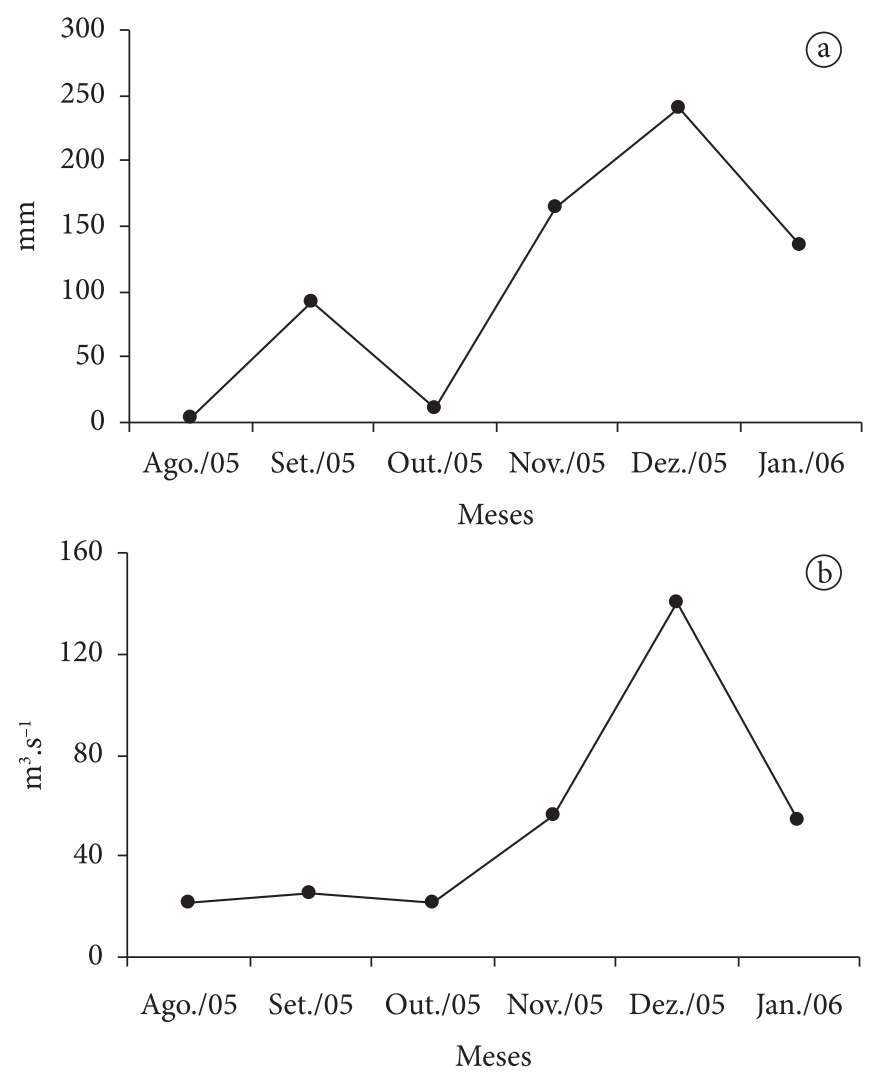

Figura 4 - Variação mensal de pluviosidade (a) e vazão (b) do Rio Muriaé entre agosto de 2005 a janeiro de 2006. (ANA/2006).

Os resultados obtidos no presente estudo são preliminares, dessa forma, torna-se necessário ampliar o número de peixes estudados por espécie e incluir coletas ao longo de todos os meses do ano, de modo que o padrão de concentração observado para o Hg seja confirmado.

Agradecimentos - Ao Dr. Paulo Buckup, especialista do Museu Nacional e ao Dr. Ronaldo Novelli que identificaram as espécies trabalhadas. Aos técnicos do LCA Marcelo Gomes de Almeida e André Luis dos Santos Machado e a aluna de I.C. Cristiane dos Santos Vergílio pelo auxílio nas análises de mercúrio. A Dra. Ana Paula Madeira DiBeneditto pela revisão e sugestões ao manuscrito. A PROEX da UENF pelo apoio financeiro a BFA (Bolsa de Extensão), ao CNPq pela bolsa de pesquisa de CEVC (proc. $n^{\circ} 306603 / 2006-3$ ) e ao Instituto Nacional de Ciência e Tecnologia de Transferência de Materiais Continente-Oceano (CNPq Proc.: 573.601/2008-9). 


\section{REFERÊNCIAS BIBLIOGRÁFICAS}

ALAIN, B., RÉGINE, M., GILLES, D. \& YANNICK, D., 2006, Mercury distribution in fish organs and food regimes: Significant elationships from twelve species collected in French Guiana (Amazonian basin). Sci. Total Environ., 368: 262-270. doi: 10.1016/j.scitotenv.2005.09.077

ALMEIDA, M. G., REZENDE, C. E. \& SOUZA, C. M. M., 2007, Variação temporal, transporte e partição de $\mathrm{Hg}$ e carbono orgânico nas frações particulada e dissolvida da coluna d'água da bacia inferior do rio Paraíba do Sul, RJ, Brasil. Geochim. Brasil, 21: 111-129.

ANA, Agência Nacional de Águas, 2006, Available from: <www. ana.gov.br>.

ANVISA, Agência Nacional de Vigilância Sanitária, 1998, Portaria 685 de 27 de Agosto de 1998: "Princípios Gerais para o Estabelecimento de Níveis Máximos de Contaminantes Químicos em Alimentos". ANVISA, Brasília, DF. Available from: <www. anvisa.gov.br/legis/resol/mercosul/alimentos/102 94>. Access in: $01 \mathrm{dez} .2006$

AZEVEDO, P., 1938. O cascudo dos açudes nordestinos "Plecostomus plecostomus". Arq. Inst. Biol., 9: 211-224.

BASTOS, W. R., MALM, O., PFEIFFER, W. C. \& CLEARY, D., 1998, Establishment and Analytical Quality Control of Laboratories for $\mathrm{Hg}$ Determination in Biological and Geological Samples in the Amazon, Brazil. Cienc. Cult., 50: 255-260.

BENEDITO-CECILIO, E. B. \& ARAÚJO-LIMA, C. A. R. M. A., 2002, Variation in the carbon isotope composition of Semaprochilodus insignis, a detritivorous fish associated with oligotrophic and eutrophic Amazonian rivers. J. Fish Biol., 60: 1603-1607. doi:10.1006/jfbi.2002.2012.

CÂMARA, V. M., 1990, O caso de Campos, R.J.: Estudo do quadro de morbidade causado pela exposição pregressa dos trabalhadores aos fungicidas mercuriais. In: Riscos e consequências do uso do mercúrio. FINEP/CNPq/MS/IBAMA, Rio de Janeiro.

CARVAlHO, C. E. V., OVALlE, A. R. C., REZENDE, C. E., SALOMÃO, M. S. M. B., MOLISANI, M. M. \& LACERDA,
L. D., 1999, Seasonal variation of particulate heavy metals in the Lower Paraíba do Sul River Drainage Basin, R.J. Brazil. Environ. Geol., 37: 297-302. doi: 10.1007/s002540050387.

DOREA, J. G., BARBOSA, A. C. \& SILVAG, S., 2006, Fish mercury bioaccumulation as a function of feeding behavior and hydrological cycles of the Rio Negro, Amazon. Comp. Biochem. Physiol., 142: 275-283.

FÖRSTNER, U. E. \& WITTMAN, G. T. W., 1983, Metal Pollution in the Aquatic Environmental. $2^{\text {th }}$ ed. Spriger-Verlag, Berlin, 486p.

PFEIFFER, W. C., MALM, O., LACERDA, L. D. \& KAREZ, C. S., 1992, Contaminação Ambiental e Humana por Metais Pesados: uma Revisão. In: T. B. Maciel, $O$ ambiente inteiro: A contribuição Crítica da Universidade na Questão Ambiental. Editora UFRJ, Rio de Janeiro.

RODRIGUES, M. T., 2003, Distribuição de Mercúrio em Hoplias malabaricus na Porção Inferior no Paraíba do Sul. Tese Universidade Estadual do Norte Fluminense - UENF, Rio de Janeiro, 49p.

SKOOG, D. A. \& LEARY, J. L., 1992, Principles of Instrumental Analysis. $4^{\text {th }} \mathrm{ed}$. Saunders College Publishing, New York, 220p.

SOUZA, C. M. M., 1994, Avaliação Ambiental dos Riscos do Mercúrio, em Áreas de Garimpo no Brasil. Tese - IBCCF, Universidade Federal do Rio de Janeiro, Rio de Janeiro, 127p.

SOUZA, P. T., 2005, Avaliação da distribuição de mercúrio total em tecido muscular da ictiofauna da lagoa de Cima e Campelo na região Norte do Estado do Rio de Janeiro. Tese - Universidade Estadual do Norte Fluminense, p. 28-56.

VARGAS, M. A. B. P. \& DAMIANCE, A. A., 2003, O processo de Formação de Comitê: A experiência do Comitê das Sub-bacias Hidrográficas dos Rios Pomba e Muriaé. In: Estado das Águas no Brasil. Agência Nacional de Água - ANP, Brasília, DF.

WEIS, M., 2004, Mercury Concentrations in fish from Canadian Great Lakes Areas Concern: an analysis of data from the Canadian Department of Environment data base. Environ. Res. 95: 341- 350. doi:10.1016/j.envres.2004.01.013 